\title{
CrystEngComm
}

Check for updates

Cite this: CrystEngComm, 2021, 23, 385

Received 28th September 2020,

Accepted 9th November 2020

DOI: $10.1039 / \mathrm{d} 0 \mathrm{ce} 01426 \mathrm{e}$

rsc.li/crystengcomm

\section{MOCVD of AIN on epitaxial graphene at extreme temperatures $\dagger$}

\author{
Anelia Kakanakova-Georgieva, (iD *a Ivan G. Ivanov, ${ }^{a}$ Nattamon Suwannaharn, (iD ab \\ Chih-Wei Hsu, ${ }^{a}$ Ildikó Cora, ${ }^{\mathrm{C}}$ Béla Pécz, ${ }^{\mathrm{C}}$ Filippo Giannazzo, (D) ${ }^{\mathrm{d}}$ \\ Davide G. Sangiovanni (D) and Gueorgui K. Gueorguiev (D) ${ }^{a}$
}

\begin{abstract}
The initial stages of metal organic chemical vapor deposition (MOCVD) of AIN on epitaxial graphene at temperatures in excess of $1200^{\circ} \mathrm{C}$ have been rationalized. The use of epitaxial graphene, in conjunction with high deposition temperatures, can deliver on the realization of nanometer thin AIN whose material quality is characterized by the appearance of luminescent centers with narrow spectral emission at room temperature. It has been elaborated, based on our previous comprehensive ab initio molecular dynamics simulations, that the impact of graphene on AIN growth consists in the way it promotes dissociation of the trimethylaluminum, $\left(\mathrm{CH}_{3}\right)_{3} \mathrm{Al}$, precursor with subsequent formation of $\mathrm{Al}$ adatoms during the initial stages of the deposition process. The high deposition temperatures ensure adequate surface diffusion of the $\mathrm{Al}$ adatoms which is an essential factor in material quality enhancement. The role of graphene in intervening with the dissociation of another precursor, trimethylgallium, $\left(\mathrm{CH}_{3}\right)_{3} \mathrm{Ga}$, has accordingly been speculated by presenting a case of propagation of ultrathin GaN of semiconductor quality. A lower deposition temperature of $1100{ }^{\circ} \mathrm{C}$ in this case has better preserved the structural integrity of epitaxial graphene. Breakage and decomposition of the graphene layers has been deduced in the case of AIN deposition at temperatures in excess of $1200^{\circ} \mathrm{C}$.
\end{abstract}

\section{Introduction}

Aluminum nitride (AlN) has an ultra-wide band gap of 6.30 eV. ${ }^{1}$ Its performance as a semiconductor material was only established with the demonstration of a $\mathrm{p}-\mathrm{i}-\mathrm{n}$ diode emitting light at about $210 \mathrm{~nm}$ in the deep $\mathrm{UV}^{2}$ With this achievement, the range of band gaps covered by group III nitrides, including InN (band gap of $0.77 \mathrm{eV}$ ) ${ }^{1}$ and GaN (band gap of $3.47 \mathrm{eV}),{ }^{1}$ extended from IR to deep UV. Group III nitrides have matured into the most valuable semiconductor alloy system for the development of short wavelength light emitting diodes and lasers which support wide-spread applications. Important applications in the fields of industry, biology, environmental sensing, and medicine, become possible as the difficult issues of epitaxial growth and doping

\footnotetext{
${ }^{a}$ Department of Physics, Chemistry and Biology (IFM), Linköping University, 581 83 Linköping, Sweden. E-mail: anelia.kakanakova@liu.se

${ }^{b}$ Nanoscience and Technology Program, Graduate School, Chulalongkorn University, Bangkok, 10330, Thailand

${ }^{c}$ Centre for Energy Research, Institute of Technical Physics and Materials Science, Konkoly-Thege M. út 29-33, Budapest, 1121, Hungary

${ }^{d}$ Consiglio Nazionale delle Ricerche, Istituto per la Microelettronica e Microsistemi, Strada VIII, n. 5, Zona Industriale, I-95121, Catania, Italy

$\dagger$ Electronic supplementary information (ESI) available. See DOI: 10.1039/ doce01426e
}

of group III nitrides are being successfully resolved by applying various alternative strategies. ${ }^{3}$

It is acknowledged that group III nitrides face their next challenge in terms of integration with graphene for the development of flexible optoelectronics, ${ }^{4}$ for making practical UV photodetector devices ${ }^{5}$ and high-performance deep UV light emitting diodes ${ }^{6}$ through direct deposition of AlN and GaN on graphene. For achieving this, fabrication of an epitaxial film of a $3 \mathrm{D}$ material with $\mathrm{sp}^{3}$ bonding on a $2 \mathrm{D}$ material with $\mathrm{sp}^{2}$ bonding must be mastered. Alongside this fundamental issue, the pragmatic aspect of preserving the structural integrity of graphene must be tackled. The metal organic chemical vapor deposition (MOCVD) of thin films of AlN and GaN of semiconductor quality is typically performed under demanding conditions. These conditions present chemically aggressive precursors such as ammonia, $\mathrm{NH}_{3}$, reacting on the substrate surface with other typical precursors, e.g., trimethylgallium, $\left(\mathrm{CH}_{3}\right)_{3} \mathrm{Ga}$, and trimethylaluminum, $\left(\mathrm{CH}_{3}\right)_{3} \mathrm{Al}$, at temperatures in excess of $1000^{\circ} \mathrm{C}$ and $1200{ }^{\circ} \mathrm{C}$, respectively. Particularly, AlN thin films of high crystalline quality can be accomplished by MOCVD at extreme temperatures of $\sim 1400{ }^{\circ} \mathrm{C} . .^{7,8}$ These high temperatures relate to the strong bonding of $2.88 \mathrm{eV}$ (66.4 kcal $\mathrm{mol}^{-1}$ ) between the $\mathrm{Al}$ and $\mathrm{N}$ atoms in the crystal lattice as compared to, e.g., the Ga-N bonding of $2.2 \mathrm{eV}$ (50.7 kcal $\left.\mathrm{mol}^{-1}\right){ }^{9}$ The MOCVD of AlN requires managing of associated 
heat and gas transport phenomena and $\left(\mathrm{CH}_{3}\right)_{3} \mathrm{Al} / \mathrm{NH}_{3}$ precursor reaction complexity. ${ }^{10}$

MOCVD of thin films and nanostructures of GaN directly on graphene has been reported at temperatures in the vicinity of $1100{ }^{\circ} \mathrm{C}$. ${ }^{4,11}$ Alternatively, optimization of the nucleation of GaN on graphene at a lower temperature of 950 ${ }^{\circ} \mathrm{C}$ has been emphasized focusing on the preservation of the structural and electronic properties of graphene, which in this particular case played also the role of highly sensitive electronic material. ${ }^{5}$ MOCVD of AlN thin films on grapheneon-sapphire at a high temperature of $1200{ }^{\circ} \mathrm{C}$ has been reported, whereby the existence of the graphene layer after deposition has been confirmed by energy-dispersive X-ray spectroscopy mapping. ${ }^{6}$ The preservation of graphene layers has also been confirmed by Raman spectroscopy and electron microscopy following the direct nucleation of AlN on graphene-on-SiC substrates, however being performed at significantly reduced temperatures of 500 to $900{ }^{\circ} \mathrm{C}^{12}$

Here, we contribute to the debate about the nucleation of AlN on epitaxial graphene by applying temperatures in excess of $1200{ }^{\circ} \mathrm{C}$. We find that the use of epitaxial graphene, in conjunction with very high deposition temperatures, delivers on realization of nanometer thin AlN whose material quality is characterized by the appearance of luminescent centers with narrow spectral emission at room temperature. Epitaxial graphene intervenes with the dissociation of the $\left(\mathrm{CH}_{3}\right)_{3} \mathrm{Al}$ precursor and assists in the formation of individual $\mathrm{Al}$ adatoms. The high deposition temperatures ensure adequate surface diffusion of the $\mathrm{Al}$ adatoms which is an essential factor in material quality enhancement. The intervention of graphene in the dissociation of another precursor, $\left(\mathrm{CH}_{3}\right)_{3} \mathrm{Ga}$, was accordingly speculated by presenting a case of propagation of ultrathin GaN of semiconductor quality.

\section{Experimental}

The MOCVD processes were performed in a horizontal-type hot-wall MOCVD reactor (GR508GFR AIXTRON) which is designed for the research and development of group III nitrides of semiconductor quality. ${ }^{7,8}$ Epitaxial graphene was fabricated on a nominally on-axis 4H-SiC (0001) substrate by a hightemperature sublimation technique, ${ }^{13}$ whereby the buffer layer can successfully be decoupled from $\mathrm{SiC}$ by hydrogen intercalation in the range of applied conditions in the MOCVD reactor. ${ }^{14}$ A reflectance map collected on a $30 \mu \mathrm{m} \times 30 \mu \mathrm{m}$ sample area representing the number of layers of as-grown epitaxial graphene is presented in the ESI $\uparrow$ Epitaxial graphene was heated in molecular hydrogen at a gas-flow rate of $25 \mathrm{slm}$ up to a temperature of $1240{ }^{\circ} \mathrm{C}$ or $1410{ }^{\circ} \mathrm{C}$. These high temperatures are typically used for the deposition of AlN films of semiconductor quality on either SiC or AlN substrates in the same MOCVD reactor. ${ }^{7,8}$ The reactor was operated at a pressure of 200 mbar. The $\left(\mathrm{CH}_{3}\right)_{3} \mathrm{Al}$ and $\mathrm{NH}_{3}$ precursors were employed at a gas-flow rate of $0.7 \mathrm{sccm}$ and $2 \mathrm{slm}$, respectively. The scheme of precursor delivery involved 3 cycles in total, each of about 3 minutes and consisting of alternating flows of $\left(\mathrm{CH}_{3}\right)_{3} \mathrm{Al}$ with $\mathrm{NH}_{3}$ followed by an extra time of 10 minutes of their joint delivery. The flow of $\mathrm{NH}_{3}$ and $\mathrm{H}_{2}$ continued during the cooling down stage of the overall MOCVD process. We also present a case of nucleation of $\mathrm{GaN}$ on epitaxial graphene. The deposition protocol involved only one cycle of alternating the flow of the $\left(\mathrm{CH}_{3}\right)_{3} \mathrm{Ga}$ precursor with $\mathrm{NH}_{3}$. The $\left(\mathrm{CH}_{3}\right)_{3} \mathrm{Ga}$ precursor was employed at a gas-flow rate of $0.442 \mathrm{sccm}$. The deposition temperature was $1100{ }^{\circ} \mathrm{C}$. A temperature of $1100{ }^{\circ} \mathrm{C}$ is typically used for the deposition of $\mathrm{GaN}$ on $\mathrm{SiC}$ substrates in the same reactor, after first depositing an AlN nucleation layer at $1240{ }^{\circ} \mathrm{C}$. A flow of $\mathrm{N}_{2}$ gas at $6 \mathrm{slm}$ was added to the flow of $\mathrm{H}_{2}$ gas for a closer match between the overall MOCVD processes of GaN on SiC and epitaxial graphene.

The morphology of the samples was evaluated by tapping mode atomic force microscopy (AFM) (VEECO, AFM Dimension 3100). The probe was a $\mathrm{Si}$ cantilever with reflective back-side $\mathrm{Al}$ coating and was commercially acquired from $\mathrm{Nu}$ Nano Ltd (SCOUT 350R). Conductive atomic force microscopy (C-AFM) current mapping and local $I-V$ measurements on the GaN-onepitaxial graphene sample were carried out using a DI3100 AFM equipped with the TUNA module. The sample surface was scanned with a Pt-coated Si tip, while a bias was applied to the sample backside.

The Raman spectra were recorded using a home-built micro-Raman setup based on a monochromator (Jobin-Yvon, model HR460) equipped with a charge-coupled device (CCD) camera. The objective lens has a magnification of $100 \times$ and numerical aperture NA $=0.95$ resulting in a $\sim 0.85 \mu$ m diameter of the laser spot focused on the sample surface. A $532 \mathrm{~nm}$ solid-state laser was used as an excitation source. The power was kept below $1 \mathrm{~mW}$ to avoid thermal damage to the sample. The spectral resolution of the system is $\sim 5.5 \mathrm{~cm}^{-1}$.

\section{Results and discussion}

The MOCVD process at $1410{ }^{\circ} \mathrm{C}$ resulted in the formation of large crystallites of AlN sparsely distributed across the surface. One such AlN crystallite is presented by its 3D topographic image in Fig. 1(a). The AlN crystallites can reach a lateral size of about $10 \mu \mathrm{m}$ and a height of about $450 \mathrm{~nm}$. The related plan-view image and height line-scan are presented in Fig. 1(c) and (e), respectively. The MOCVD process at $1240{ }^{\circ} \mathrm{C}$ resulted in the formation of individual crystallites together with larger areas of lateral coalescence, Fig. 1(b) and (d). In this case the height of the AlN crystallites was limited to about 125 nm, Fig. 1(f). The AlN has an epitaxial relationship with the SiC substrate whereby high-resolution TEM images of the $\mathrm{AlN} / \mathrm{SiC}$ interface and associated selected area electron diffraction patterns are shown in the ESI. +

It might be argued that features of self-assembly of AlN crystallites resemble those observed for self-assembled GaN crystallites on graphene, as reported in ref. 5. An assumption to explain the similarities is that the low surface energy of graphene impedes nucleation of AlN on graphene, as indicated by the low density of nuclei. On the other hand, the low barrier for metal migration on graphene $e^{15,16}$ 

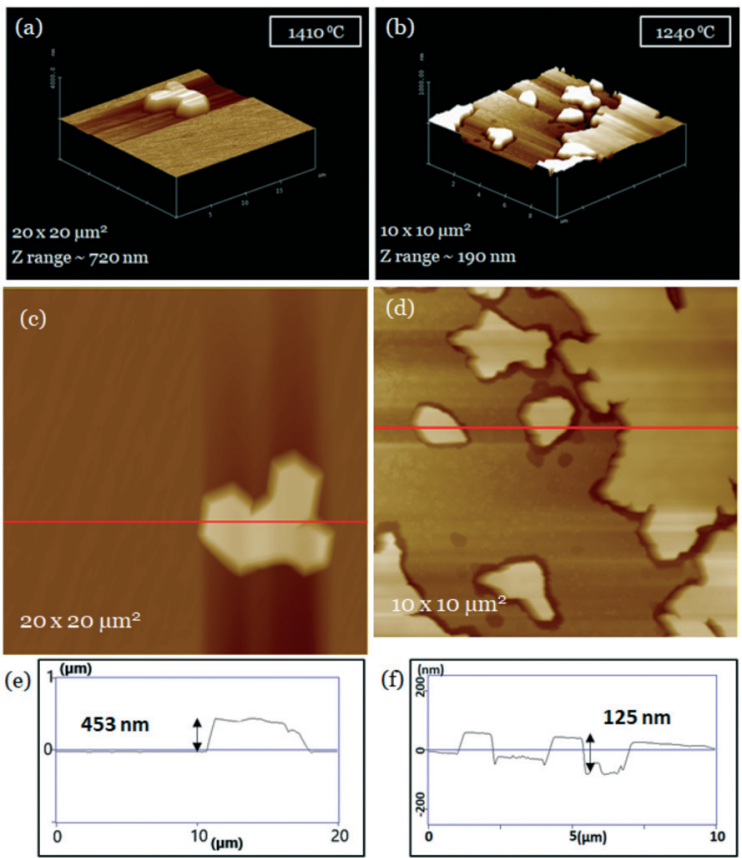

Fig. 1 AFM 3D topographic images of AIN crystallites and coalesced areas deposited at $1410{ }^{\circ} \mathrm{C}$ (a) and $1240{ }^{\circ} \mathrm{C}$ (b). The dark-brown areas in the vicinity of the AIN crystallites, appearing to be a basin, are artifacts caused by the drastic change in height along the scanning direction. Plan-view images of the AIN crystallites and coalesced areas deposited at $1410{ }^{\circ} \mathrm{C}$ (c) and $1240{ }^{\circ} \mathrm{C}$ (d). The cross-sectional linescans are correspondingly shown in (e) and (f).

promotes adatom diffusion ( $\mathrm{Al}$ adatoms in this case $^{17}$ ) and lateral growth.

The larger degree of coverage with AlN crystallites and larger areas of coalescence make Raman and photoluminescence (PL) mapping on the sample deposited at $1240{ }^{\circ} \mathrm{C}$ possible, Fig. 2 . The characteristic $\mathrm{E}_{2}$ (high) phonon mode of AlN is expected at $\sim 660 \mathrm{~cm}^{-1}$. However, its direct observation in the Raman spectra is deemed difficult due to (i) the expected weak signal owing to the limited thickness of the AlN crystallites and, hence, the small volume excited by the laser; and (ii) the rather strong background signal from second-order Raman bands of the SiC substrate in this spectral region. While the Raman signature of AlN cannot be discerned, the presence of AlN is manifested by a weak broadband PL background on which occasionally rather narrow peaks appear during the mapping; these are marked with their peak wavelengths in Fig. 2. The appearance of these peaks is well localized only to certain positions on the sample. This circumstance suggests that they are most likely due to point defects in AlN, possibly single-photon emitters, analogous to those recently observed in AlN films. ${ }^{18,19}$ Similar single-photon emitters have been observed also in $\mathrm{GaN}^{20}$ We notice that the sole experimental studies on emission from point defects in AlN are reported in ref. 18 and 19 to the best of our knowledge. The peak positions as observed in our work (Fig. 2) are consistent with those listed in the table for AlN in the latter reference. However, neither

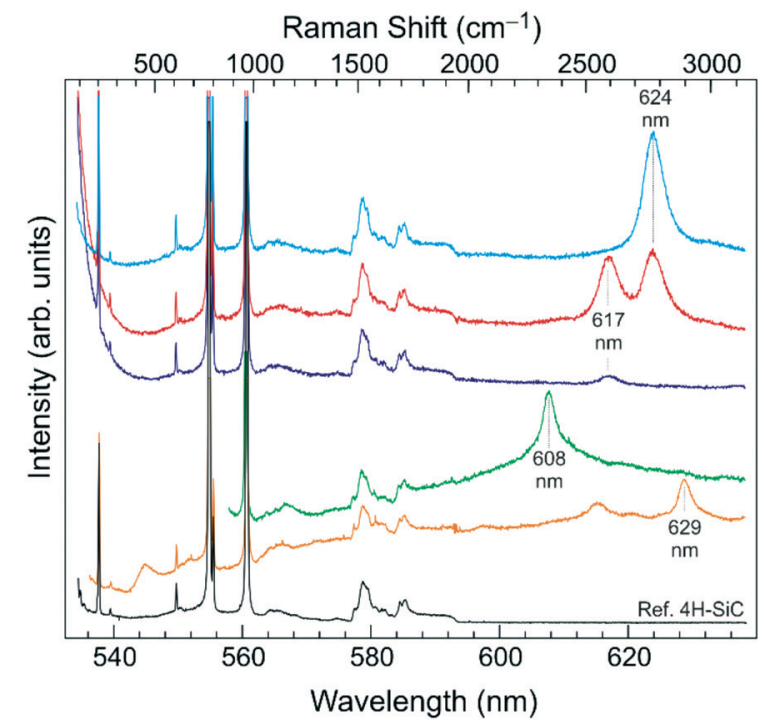

Fig. 2 Spectra from different spots on the AIN crystallites deposited at $1240{ }^{\circ} \mathrm{C}$ (top three curves). The fourth and fifth curves from the top represent spectra at different spots on another AIN sample deposited at $1240{ }^{\circ} \mathrm{C}$ by applying a larger flow of $\mathrm{NH}_{3}$ of $7 \mathrm{slm}$. The reference Raman spectrum of $4 \mathrm{H}-\mathrm{SiC}$ showing the first and second order Raman contributions of the substrate spectrum in the remaining spectra is shown at the bottom. The top scale on the horizontal axis is given in Raman shifts to help identify the Raman peaks from the substrate.

ref. 19 nor the present work examines the observed PL lines for single photon emission; this is done in ref. 18. Thus, we associate the observed peaks with luminescence from unidentified point defects in AlN. We note that the fourth and fifth spectra from the top in Fig. 2 were obtained from another AlN sample deposited at $1240{ }^{\circ} \mathrm{C}$ by applying a larger flow of $\mathrm{NH}_{3}$ of $7 \mathrm{slm}$. In this case, the peaks were centered at wavelengths of 608 and 629 nm, Fig. 2.

Although in our case the observed lines serve merely as an indicator of the presence of AlN, it is tempting to reflect on investigations into AlN as a host material for the realization of single-photon emission from point defects. Primarily, advanced first-principles calculations have been performed to identify point defects and defect complexes in AlN as candidates for solid-state qubits. ${ }^{21}$ Recently, first-principles calculations based on density functional theory have been employed to rationalize first-time experimentally detected single-photon emissions from point defects in AlN films. ${ }^{18}$ The spectra of the observed single-photon emissions ranged from visible to near-infrared, 550 to $1000 \mathrm{~nm}$, and have been reported to originate from point defects in $2 \mu \mathrm{m}$ thick AlN films grown by MOCVD. In that work, ${ }^{18}$ high quality AlN films were deposited after an elaborate preparation of nanopatterned sapphire substrates via nanoimprint lithography. It is acknowledged that resolving material quality issues can deliver much-needed experimental verification of single-photon emission at room temperature. ${ }^{22}$

In this study, we report the results pertaining to the observation of luminescence from point defects in AlN crystallites with a thickness of only $125 \mathrm{~nm}$. The use of 
epitaxial graphene, in conjunction with a very high deposition temperature of $1240{ }^{\circ} \mathrm{C}$, can thus deliver on the realization of nanometer thin $\mathrm{AlN}$ of apparently good material quality. While we refer to the formation of AlN crystallites on epitaxial graphene at temperatures in excess of $1200{ }^{\circ} \mathrm{C}$, the Raman spectra of the samples deposited at 1240 and $1410{ }^{\circ} \mathrm{C}$ did not display the characteristic modes of graphene. It is therefore inferred that the structural integrity of graphene cannot withstand the overall duration of application of the $\left(\mathrm{CH}_{3}\right)_{3} \mathrm{Al}$ precursor for the deposition of AlN at these extreme temperatures. However, the impact of graphene on AlN growth consists, for example, in the way it promotes dissociation of the $\left(\mathrm{CH}_{3}\right)_{3} \mathrm{Al}$ precursor with subsequent formation of $\mathrm{Al}$ adatoms at the initial stages of the deposition process. Atomistic- and electronic-level understanding of $\left(\mathrm{CH}_{3}\right)_{3} \mathrm{Al} /$ graphene surface reactions has previously been achieved by our comprehensive $a b$ initio molecular dynamics (AIMD) simulations. ${ }^{17}$

AIMD simulations point to the formation of individual $\mathrm{Al}$ adatoms of long lifetime and surface diffusivity, which could act as sites for AlN nucleation on graphene. ${ }^{17,23}$ Building on the understanding for the dissociative adsorption of the $\left(\mathrm{CH}_{3}\right)_{3} \mathrm{Al}$ precursor, our present deposition procedure of AlN on graphene has been designed (specifically, by firstly introducing the $\left(\mathrm{CH}_{3}\right)_{3} \mathrm{Al}$ precursor into the reactor) in order to optimize the experimental conditions for growth of AlN of recognized material quality. Indeed, AIMD indicates that pristine graphene is essentially inert toward reactions with ammonia. Nevertheless, the formation of $\mathrm{Al}$ (and $\mathrm{C}$ ) adatoms, consequent to $\left(\mathrm{CH}_{3}\right)_{3} \mathrm{Al} /$ graphene collisions, provides surface sites of higher reactivity which may facilitate $\mathrm{NH}_{3}$ dissociation and thus open a channel for the delivery of $\mathrm{N}$ atoms to the graphene surface. ${ }^{17}$

The formation of $\mathrm{C}$ adatoms might be expected to significantly affect the local reactivity and structural stability of graphene, especially at high temperatures. The $\mathrm{C}$ adatom dynamics on graphene, by means of surface migration and occurrence of exchange reactions, ${ }^{17}$ is expected to contribute to weakening of the $\mathrm{C}$ bonds vicinal to pre-existing defects of the graphene lattice, e.g., Stone-Wales, pentagonal, and double pentagon defects, thus assisting the formation of holes in the bonding network. Consequently, the increased reactivity of unsaturated bonds in graphene, assisted by attachment of gas radical molecules, may cause partial or complete decomposition of the graphene layers. Hence, breakage and decomposition of the graphene layers originate in the initial dissociation of the $\left(\mathrm{CH}_{3}\right)_{3} \mathrm{Al}$ precursor, while the extent of the decomposition depends strongly on the deposition temperature.

It is worth emphasizing that the formation of individual $\mathrm{Al}$ adatoms on graphene, being a direct consequence of the dissociation of the $\left(\mathrm{CH}_{3}\right)_{3} \mathrm{Al}$ precursor itself, ${ }^{17}$ can rationalize the material quality of the accomplished nanometer thin AlN. The formation of individual $\mathrm{Al}$ adatoms, which can rapidly diffuse on graphene, ${ }^{17}$ is a plausible mechanism for the initial nucleation of AlN, provided that the presence of $\mathrm{Al}$ (and C) adatoms promotes dissociation of $\mathrm{NH}_{3}$ on graphene with subsequent release of nitrogen atoms. By comparison, the MOCVD of AlN on bare SiC (and other substrates) is dominated by the initial formation in the gas phase and/or near the hot substrate surface of certain $\left(\mathrm{CH}_{3}\right)_{2} \mathrm{AlNH}_{2}$ species with direct $\mathrm{Al}-\mathrm{N}$ bonding as a result of the $\left(\mathrm{CH}_{3}\right)_{3} \mathrm{Al}$ precursor interaction with $\mathrm{NH}_{3} \cdot{ }^{10,24}$ At the surface, these species can ultimately break down into dimers carrying $\mathrm{Al}-\mathrm{N}$ bonds. Alternatively, if the $\left(\mathrm{CH}_{3}\right)_{3} \mathrm{Al}$ dissociation is presented as a sequential elimination of methyl groups in the gas phase, ${ }^{9,24}$ the elimination of the last methyl group, considering the strong $\mathrm{Al}-\mathrm{C}$ bond of $\sim 82 \mathrm{kcal} \mathrm{mol}^{-1},{ }^{25}$ can only occur by adsorption on the surface upon interaction with available $\mathrm{NH}_{x}$ species which may result from the chemisorption of the very strongly bonded $\mathrm{NH}_{3}$ precursor. ${ }^{17,25}$ Thus, the nucleation of $\mathrm{AlN}$ on $\mathrm{SiC}$ is governed by surface adsorption and decomposition of gas species upon the formation of $\mathrm{Al}-\mathrm{N}$ bonds, unlike the case of nucleation of AlN on epitaxial graphene, which is governed by individual mobile $\mathrm{Al}$ adatoms reaching favorable nucleation sites.

It is to be assumed that graphene can also intervene in a similarly specific way, at the atomistic level, in the dissociation of the $\left(\mathrm{CH}_{3}\right)_{3} \mathrm{Ga}$ precursor, which is applied in the MOCVD of GaN. Formation of individual Ga adatoms, consequent to $\left(\mathrm{CH}_{3}\right)_{3} \mathrm{Ga} /$ graphene surface reactions, can thus be suggested to initiate direct nucleation of $\mathrm{GaN}$ on epitaxial graphene.

A typical tapping mode AFM morphology and the corresponding phase map of GaN deposited on graphene at a temperature of $1100{ }^{\circ} \mathrm{C}$ are presented in Fig. 3(a) and (b), respectively. A relatively smooth morphology with a low RMS roughness of $0.68 \mathrm{~nm}$ was deduced from the topographic
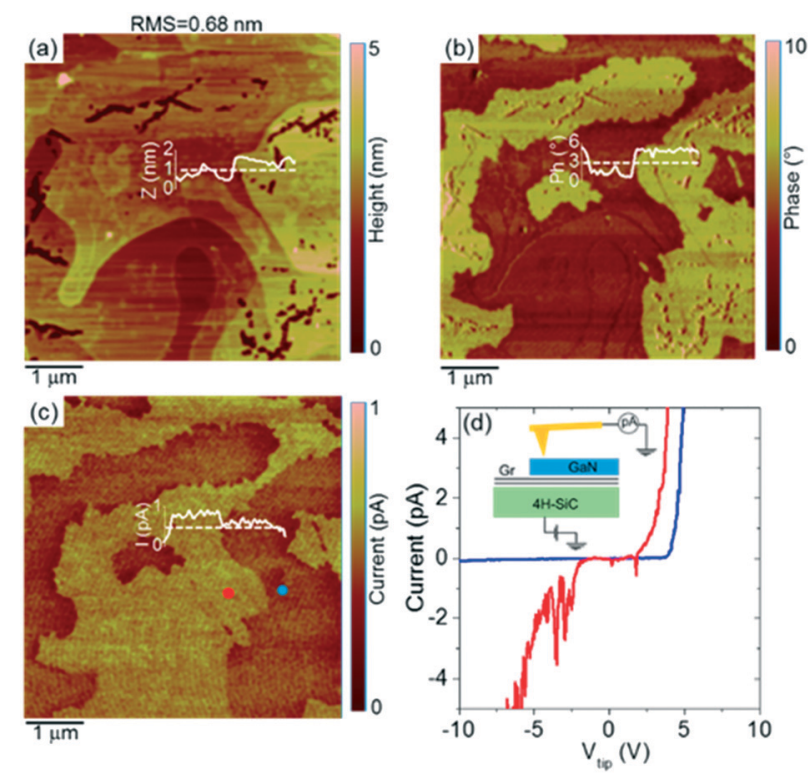

Fig. 3 Tapping mode AFM morphology (a) and phase (b) images of ultrathin $\mathrm{GaN}$ deposited at $1100^{\circ} \mathrm{C}$ on epitaxial graphene. (c) C-AFM current map acquired in the same sample area. Height, phase, and current line-scans are shown in the insers of panels (a)-(c). (d) Local current-voltage characteristics acquired at the positions indicated in (c). 
map. The phase map exhibits regions with significantly different contrast (bright and dark areas), indicating a different electrostatic interaction between the tip and the surface associated with an inhomogeneous GaN coverage. From the comparison between the height and phase linescans in the insets of Fig. 3(a) and (b), it can be deduced that the bright phase contrast is associated with areas covered by ultrathin GaN domains, whereas the dark phase contrast is associated with bare epitaxial graphene areas. This is further confirmed by local current mapping (see Fig. 3(c)) performed in the same sample region by conductive atomic force microscopy (C-AFM) in the vertical (i.e., front-to-back) configuration (as depicted in the inset of Fig. 3(d)).

A reduced vertical current is clearly observed in the epitaxial graphene regions covered by ultrathin GaN with respect to the uncovered ones. Fig. 3(d) indicates two representative local current-voltage $(I-V)$ analyses acquired in the GaN covered and bare epitaxial graphene areas (positions indicated by the blue and red points in Fig. 3(c)). A rectifying behaviour, dominated by the tip/ GaN Schottky contact, ${ }^{26}$ is observed in the GaN covered regions, indicating a good semiconducting quality of GaN. On the other hand, a more symmetric behaviour is observed in the uncovered regions, probably dominated by the graphene/SiC Schottky contact. ${ }^{27}$ It is worth mentioning that the C-AFM technique has been employed in the past to investigate the electrical quality of $\mathrm{GaN}$ materials. ${ }^{28}$ In the case of $\mathrm{GaN}$ on a foreign substrate, current maps and local $I-V$ characteristics measured by the C-AFM metal tip provided information not only on the Schottky barrier with the GaN surface, but also on the presence of electrically active defects, such as threading dislocations connecting the GaN surface with the substrate. ${ }^{29}$ In the present case of ultrathin GaN grown by MOCVD on epitaxial graphene, the local $I-V$ characteristics demonstrate the formation of a Schottky contact between the metal tip and the GaN covered region. In particular, the extremely low leakage current measured under reverse bias polarization is consistent with the wide bandgap of the GaN material. Furthermore, the uniform contrast observed in the current map of the GaN covered areas indicates the absence of conductive defects through ultrathin GaN.

It is to be noted that the epitaxial growth of $\mathrm{GaN}$ on $\mathrm{SiC}$ typically requires the deposition of an AlN nucleation layer, which in this study has been eliminated with the employment of a $\mathrm{SiC}$ substrate terminated with epitaxial graphene. Graphene did sustain better structural integrity against the conditions which are characteristic of the MOCVD of GaN performed at a temperature of $1100{ }^{\circ} \mathrm{C}$. Raman spectra, using the $532 \mathrm{~nm}$ laser excitation, indicate the preservation of the graphene layers at the $\mathrm{GaN} / \mathrm{SiC}$ interface, (see the ESI†े). Indication for the ultrathin GaN may be obtained by using UV excitation for reducing the $\mathrm{SiC}$ contribution to the Raman spectra and recording the phonon mode $A_{1}(\mathrm{LO})$ in $\mathrm{GaN}^{30}$ A case of ultrathin $\mathrm{GaN}$ imaged using an in-lens detector for collection of secondary electrons is presented in the ESI. $\dagger$

\section{Conclusions}

The use of epitaxial graphene, in conjunction with high deposition temperatures of 1240 and $1410{ }^{\circ} \mathrm{C}$, can deliver on the realization of nanometer thin AlN whose material quality is characterized by the appearance of luminescent centers with narrow spectral emission at room temperature. It has been elaborated, based on our previous comprehensive AIMD simulations, that the impact of graphene on AlN growth consists in the way it promotes the dissociation of the $\left(\mathrm{CH}_{3}\right)_{3}$ $\mathrm{Al}$ precursor with subsequent formation of $\mathrm{Al}$ adatoms at the initial stages of the deposition process. The high deposition temperatures ensure adequate surface diffusion of the $\mathrm{Al}$ adatoms which is an essential factor in material quality enhancement. The role of graphene in intervening with the dissociation of another precursor, $\left(\mathrm{CH}_{3}\right)_{3} \mathrm{Ga}$, was accordingly speculated by presenting a case of propagation of ultrathin $\mathrm{GaN}$ of semiconductor quality. The results of this work suggest that surface dissociation of $\left(\mathrm{CH}_{3}\right)_{3} \mathrm{Al}$ and $\left(\mathrm{CH}_{3}\right)_{3} \mathrm{Ga}$, combined with elevated deposition temperatures, is responsible for the decomposition of the graphene layers. However, the extent of the decomposition depends strongly on the deposition temperature. Our results imply that the structural integrity of graphene can be better preserved at a deposition temperature of $1100{ }^{\circ} \mathrm{C}$ in the MOCVD of GaN and it cannot withstand the overall duration of application of the $\left(\mathrm{CH}_{3}\right)_{3} \mathrm{Al}$ precursor for the deposition of AlN at temperatures in excess of $1200{ }^{\circ} \mathrm{C}$.

\section{Conflicts of interest}

There are no conflicts to declare.

\section{Acknowledgements}

The authors thank Dr. T. Iakimov and Prof. R. Yakimova for kindly providing samples of epitaxial graphene. The authors thank N. Szász and L. Illés for TEM specimen preparation. This work was supported by the FLAG-ERA 2015 JTC project GRIFONE through the Swedish Research Council VR201506816 (A. Kakanakova, project leader GRIFONE), the National Research Development and Innovation Office, Hungary, NN 118914 (B. Pécz), and in-kind resources of CNR-IMM, Italy. A. K.-G. acknowledges support through projects VR2017-04071 and ÅF15-557. G. K. G. acknowledges support through project ÅF18-266. G. K. G. and D. G. S. acknowledge resources provided by the Swedish National Infrastructure for Computing (SNIC) at the National Supercomputer Center (NSC) in Linköping (SNIC 2020/5-146 and SNIC 2020/14-17) partially funded by the Swedish Research Council through grant agreement VR2018-05973. D. G. S. acknowledges financial support from the Olle Engkvist Foundation. I. G. I. acknowledges support through project VR2016-05362. 


\section{References}

1 Y.-C. Tsai and C. Bayram, Sci. Rep., 2019, 9, 6583.

2 Y. Taniyasu, M. Kasu and T. Makimoto, Nature, 2006, 441, 325.

3 M. Kneissl, T.-Y. Seong, J. Han and H. Amano, Nat. Photonics, 2019, 13, 233.

4 J. Kim, C. Bayram, H. Park, C.-W. Cheng, C. Dimitrakopoulos, J. A. Ott, K. B. Reuter, S. W. Bedell and D. K. Sadana, Nat. Commun., 2014, 5, 4836.

5 T. Journot, V. Bouchiat, B. Gayral, J. Dijon and B. Hyot, ACS Appl. Mater. Interfaces, 2018, 10, 18857.

6 Z. Chen, Z. Liu, T. Wei, S. Yang, Z. Dou, Y. Wang, H. Ci, H. Chang, Y. Qi, J. Yan, J. Wang, Y. Zhang, P. Gao, J. Li and Z. Liu, Adv. Mater., 2019, 1807345.

7 M. Feneberg, N. T. Son and A. Kakanakova-Georgieva, Appl. Phys. Lett., 2015, 106, 242101.

8 D. Nilsson, E. Janzén and A. Kakanakova-Georgieva, J. Phys. D: Appl. Phys., 2016, 49, 175108.

9 S. Keller and S. P. DenBaars, J. Cryst. Growth, 2003, 248, 479.

10 R. B. dos Santos, R. Rivelino, F. de Brito Mota, G. K. Gueorguiev and A. Kakanakova-Georgieva, J. Phys. D: Appl. Phys., 2015, 48, 295104.

11 G. Sarau, M. Heilmann, M. Bashouti, M. Latzel, C. Tessarek and S. Christiansen, ACS Appl. Mater. Interfaces, 2017, 9, 10003.

12 Z. Y. Al Balushi, T. Miyagi, Y.-C. Lin, K. Wang, L. Calderin, G. Bhimanapati, J. M. Redwing and J. A. Robinson, Surf. Sci., 2015, 634, 81.

13 R. Yakimova, Ch. Virojanadara, D. Gogova, M. Syväjärvi, D. Siche, K. Larsson and L. Johansson, Mater. Sci. Forum, 2010, 645-648, 565.

14 I. Shtepliuk, I. G. Ivanov, T. Iakimov, R. Yakimova, A. Kakanakova-Georgieva, P. Fiorenza and F. Giannazzo, Mater. Sci. Semicond. Process., 2019, 96, 145.

15 M. Manadé, F. Viñes and F. Illas, Carbon, 2015, 95, 525.

16 A. Jamnig, D. G. Sangiovanni, G. Abadias and K. Sarakinos, Sci. Rep., 2019, 9, 6640.

17 D. G. Sangiovanni, G. K. Gueorguiev and A. KakanakovaGeorgieva, Phys. Chem. Chem. Phys., 2018, 20, 17751.
18 Y. Xue, H. Wang, N. Xie, Q. Yang, F. Xu, B. Shen, J. Shi, D. Jiang, X. Dou, T. Yu and B. Sun, J. Phys. Chem. Lett., 2020, 11, 2689.

19 R. Lin, Single Defects in III-Nitrides as Promising Candidates for Monochromatic Single-Photon Sources, Master thesis, Linköping University, LITH-IFM-A-EX-16/3256-SE, 2016.

20 A. M. Berhane, K.-Y. Jeong, Z. Bodrog, S. Fiedler, T. Schröder, N. V. Triviño, T. Palacios, A. Gali, M. Toth, D. Englund and I. Aharonovich, Adv. Mater., 2017, 29, 1605092.

21 J. B. Varley, A. Janotti and C. G. Van de Walle, Phys. Rev. B, 2016, 93, 161201(R).

22 M. J. Holmes, K. Choi, S. Kako, M. Arita and Y. Arakawa, Nano Lett., 2014, 14, 982.

23 A. Kakanakova-Georgieva, G. K. Gueorguiev, D. G. Sangiovanni, N. Suwannaharn, I. G. Ivanov, I. Cora, B. Pécz, G. Nicotra and F. Giannazzo, Nanoscale, 2020, 12, 19470.

24 T. G. Mihopoulos, V. Gupta and K. F. Jensen, J. Cryst. Growth, 1998, 195, 733.

25 R. B. dos Santos, R. Rivelino, F. de Brito Mota, A. Kakanakova-Georgieva and G. K. Gueorguiev, Dalton Trans., 2015, 44, 3356.

26 F. Giannazzo, F. Roccaforte, F. Iucolano, V. Raineri, F. Ruffino and M. G. Grimaldi, J. Vac. Sci. Technol., B, 2009, 27, 789.

27 F. Giannazzo, I. Shtepliuk, I. G. Ivanov, T. Iakimov, A. Kakanakova-Georgieva, E. Schilirò, P. Fiorenza and R. Yakimova, Nanotechnology, 2019, 30, 284003.

28 F. Iucolano, F. Roccaforte, F. Giannazzo and V. Raineri, J. Appl. Phys., 2007, 102, 113701.

29 F. Giannazzo, G. Fisichella, G. Greco, P. Fiorenza and F. Roccaforte, Conductive atomic force microscopy of twodimensional electron systems: from AlGaN/GaN heterostructures to graphene and $\mathrm{MoS} 2$, in Conductive Atomic Force Microscopy: Applications in Nanomaterials, ed. M. Lanza, Wiley-VCH, 2017, pp. 163-182.

30 Z. Y. Al Balushi, K. Wang, R. K. Ghosh, R. A. Vilá, S. M. Eichfeld, J. D. Caldwell, X. Qin, Y.-C. Lin, P. A. DeSario, G. Stone, S. Subramanian, D. F. Paul, R. M. Wallace, S. Datta, J. M. Redwing and J. A. Robinson, Nat. Mater., 2016, 15, 1166, DOI: $10.1038 /$ nmat4742, ESI $\dagger$. 